\title{
Automedicação entre acadêmicos de enfermagem: pesquisa-ação em uma instituição privada em Rondônia
}

\author{
Self-medication among nursing students: action research in a private institution in Rondônia \\ La automedicación en estudiantes de enfermería: investigación-acción en una institución privada \\ en Rondônia
}

Andrezza Iara Rodrigues Vieira ORCID: https://orcid.org/0000-0002-7047-0871 Faculdade Interamericana de Porto Velho, Brasil E-mail: andrezza.yara@gmail.com

Vanessa de Souza Canterle

ORCID: https://orcid.org/0000-0002-3891-986X Faculdade Interamericana de Porto Velho, Brasil E-mail: vanessa_canterle@ @otmail.com

Bruno Gomes Brumado ORCID: https://orcid.org/0000-0001-7752-7335 Faculdade Interamericana de Porto Velho, Brasil E-mail: brunobrumado7@gmail.com

Ivana Annely Cortez da Fonseca ORCID: https://orcid.org/0000-0001-6598-5945 Faculdade Interamericana de Porto Velho, Brasil E-mail: ivana.fonseca@uniron.edu.br

Flávia Rodrigues de Araújo Durães ORCID: https://orcid.org/0000-0002-7519-508X Faculdade Interamericana de Porto Velho, Brasil

E-mail: flaviaaraujo.rodrigues@gmail.com

Keilla dos Santos Andrade ORCID: https://orcid.org/0000-0001-5554-4310 Faculdade Interamericana de Porto Velho, Brasil E-mail: keilla16amdrade@gmail.com

\begin{abstract}
Resumo
Introdução: A automedicação constitui-se uma prática perigosa, devido seus malefícios para a saúde. Estudos sobre a automedicação entre acadêmicos de enfermagem pode fomentar a educação em saúde e práticas intervencionistas na graduação. Objetivo: Realizar uma oficina pedagógica, como forma de intervenção, voltada para a educação em saúde sobre a prática da automedicação entre os acadêmicos de enfermagem em uma instituição de ensino superior. Métodos: Estudo de abordagem qualitativa do tipo pesquisa-ação utilizando como base a teoria da problematização o arco de Charles Maguerez. A amostra do estudo foi composta por 21 acadêmicos de enfermagem de uma instituição de ensino superior. Resultados: Observou-se que a maioria dos acadêmicos faz uso de medicamentos sem prescrição médica, dentre os sintomas que os levam a ingerir medicamentos elenca-se dores de cabeça, dores musculares, gripes, inflamações, tosse, azia, febre e cólica menstrual, sendo que as classes medicamentosas mais utilizadas são analgésicos, anti-inflamatórios e antitérmicos. A autoconfiança em se automedicar está fortemente relacionada ao fato de já terem cursado a disciplina de terapia medicamentosa, apesar de estarem cientes dos riscos, fato que corrobora com a prática de recomendações para familiares e amigos. Considerações Finais: $\mathrm{O}$ estudo forneceu informações e conhecimentos relevantes em relação ao fenômeno da automedicação entre acadêmicos de enfermagem, após a oficina os acadêmicos demonstraram interesse em suspender a prática da automedicação, além de repassar os conhecimentos adquiridos para familiares e amigos.
\end{abstract}

Palavras-chave: Automedicação; Enfermagem; Acadêmicos; Pesquisa-ação.

\begin{abstract}
Introduction: Self-medication is a dangerous practice due to its harmful effects on health. Studies on self-medication among nursing students can foster health education and interventionist practices in undergraduate courses. Objective: Conduct a pedagogical workshop, as a form of intervention, aimed at health education on the practice of selfmedication among nursing students in a higher education institution. Methods: Study of qualitative approach of the research-action type using as basis the theory of problematization the arch of Charles Maguerez. The study sample consisted of 21 nursing students from a higher education institution. Results: It was observed that most students use
\end{abstract}


over-the-counter medications, among the symptoms that lead them to take medications are headaches, muscle pain, flu, inflammation, cough, heartburn, fever and menstrual cramps, the most used drug classes are analgesics, antiinflammatory and antipyretics. Self-confidence in self-medicating is strongly related to the fact that they have already taken the course of drug therapy, despite being aware of the risks, a fact that corroborates the practice of recommendations for family and friends. Final Considerations: The study provided relevant information and knowledge regarding the phenomenon of self-medication among nursing students, after the workshop, the students showed interest in suspending the practice of self-medication, in addition to passing on the knowledge acquired to family and friends.

Keywords: Self-medication; Nursing; Academic; Action Research.

\section{Resumen}

Introducción: La automedicación es una práctica peligrosa por sus efectos nocivos para la salud. Los estudios sobre automedicación entre estudiantes de enfermería pueden fomentar la educación para la salud y las prácticas intervencionistas en los cursos de pregrado. Objetivo: Realizar un taller pedagógico, como forma de intervención, dirigido a la educación en salud sobre la práctica de la automedicación en estudiantes de enfermería de una institución de educación superior. Métodos: Estudio de abordaje cualitativo del tipo investigación-acción tomando como base la teoría de la problematización del arco de Carlos Maguerez. La muestra del estudio estuvo constituida por 21 estudiantes de enfermería de una institución de educación superior. Resultados: Se observó que la mayoría de los estudiantes utilizan medicamentos de venta libre, entre los síntomas que los llevan a tomar medicamentos se encuentran dolores de cabeza, dolores musculares, gripe, inflamación, tos, ardor de estómago, fiebre y cólicos menstruales, las clases de drogas más utilizadas son analgésicos, antiinflamatorios y antipiréticos. La autoconfianza en la automedicación está fuertemente relacionada con el hecho de que ya hayan realizado el curso de la farmacoterapia, a pesar de ser conscientes de los riesgos, hecho que corrobora la práctica de recomendaciones para familiares y amigos. Consideraciones finales: El estudio proporcionó información y conocimientos relevantes sobre el fenómeno de la automedicación entre estudiantes de enfermería, luego del taller, los estudiantes mostraron interés en suspender la práctica de la automedicación, además de transmitir los conocimientos adquiridos a familiares y amigos.

Palabras clave: Automedicación; Enfermería; Académica; Investigación-acción.

\section{Introdução}

A automedicação tem como objetivo a busca pelo alívio de dores, desconfortos, tratamento de doenças e sintomas sem a prescrição de um profissional de saúde. Tal prática tem sido considerada um fenômeno crescente entre a população brasileira e pode estar relacionada a diferentes causas, como a facilidade de comercialização, comodidade, influência de familiares, amigos, farmacêuticos, além dos conhecimentos obtidos através da variedade de informações disponíveis (Rivera et al, 2021).

Entre profissionais e acadêmicos de enfermagem, o consumo de medicamentos é uma prática bem presente, tendo como um fator de risco para tal ação, os conhecimentos prático e teórico sobre os fármacos adquiridos na vida acadêmica e profissional (Ribeiro et al., 2018).

De acordo com Alves et al. (2019), os principais motivos que levam os acadêmicos de enfermagem a se automedicarem são experiências prévias com o sintoma ou a doença, a falta de acessibilidade aos serviços de saúde, a limitação de recursos financeiros e a indisponibilidade de tempo para buscar auxílio médico.

No estudo realizado por Gama e Secolli (2017), entre estudantes de enfermagem do estado do Amazonas, foram relacionados os problemas de saúde que levaram a prática da automedicação, os quais incluíram cólicas menstruais, dores de cabeça e abdominais. Outras causas apontadas pelos acadêmicos foram infecções de garganta e urinária, resfriado, febre, problemas gastrintestinais.

Pinto et al. (2021) discorrem sobre os riscos que a automedicação pode ocasionar, tais como: mascaramento do diagnóstico da doença, resistência medicamentosa principalmente quando relacionados ao uso de antibióticos, potenciais interações medicamentosas, alergias, intoxicação e óbito. Segundo Xavier e Silva (2021) o fácil acesso pela população às substâncias como os analgésicos, xaropes e anti-inflamatórios podem acarretar inúmeras consequências através do uso indiscriminado, elenca-se: dependência, sonolência e reações alérgicas.

Conforme a Agência Nacional de Vigilância Sanitária - ANVISA (2016), há uma lista de medicamentos isentos de prescrição, como: antitérmicos, antiácidos, analgésicos, anti-inflamatórios, descongestionantes nasais, expectorantes e 
mucolíticos. Esses medicamentos não possuem tarjas preconizadas nos medicamentos sujeitos a prescrição (tarja vermelha) ou sujeitos a controle especial (tarja preta).

A prática da automedicação é realizada por grande parte da população, desde leigos a profissionais da saúde, fazendose necessário abordar de forma elucidativa os riscos desta prática, assim como, ressaltar a importância do uso responsável de medicamentos ainda no período de graduação, para promoção de orientações educativas e utilização do fármaco de acordo com as condições clínicas, dosagem conforme a necessidade e tempo adequados, evitando-se, dessa forma, ocasionar prejuízos (Souza \& Lima, 2019).

Com a abordagem da automedicação entre os graduandos de cursos da área da saúde, espera-se que em sua prática diária tenham uma postura condizente com sua formação profissional, estando preparados para realizar orientações quanto ao uso responsável de medicamentos e que estejam aptos para utilizarem os fármacos de maneira adequada. Em vista disso, a pesquisa sobre a prática da automedicação pode reconhecer a necessidade de educar e implementar intervenções ainda em fase de graduação (Tognolli et al., 2019).

Somando-se as reflexões iniciais acerca do tema de pesquisa proposto na revisão de literatura, surgiu a seguinte questão norteadora: O que influencia a prática da automedicação entre os acadêmicos de enfermagem? Como a academia em enfermagem pode contribuir para evitar a automedicação neste grupo específico?

Nesta perspectiva, o estudo tem por objetivo elaborar uma intervenção de enfermagem sobre a prática da automedicação entre acadêmicos de enfermagem em uma Instituição de Ensino Superior em Rondônia.

\section{Metodologia}

Estudo qualitativo do tipo pesquisa-ação utilizou-se como base teórica a problematização do arco de Charles Maguerez, baseado em cinco etapas: observação da realidade; investigação dos pontos-chave; teorização; hipótese de solução e retorno à realidade (Silva et al., 2020).

Para o desenvolvimento da pesquisa foram observados os pressupostos contidos na Resolução 466/12 do Conselho Nacional de Saúde/ Ministério da Saúde, que aprova as diretrizes e normas regulamentadoras de pesquisas envolvendo seres humanos. A pesquisa iniciou-se mediante autorização do Diretor da Instituição de Ensino Superior e aprovação pelo Comitê de Ética e Pesquisa (CEP) da União Educacional do Norte Ltda (UNINORTE) em 13 (treze) de julho de 2021, sob o parecer ${ }^{\circ}$ 4.843.935 e CAAE: 48110621.0 .0000 .802$.

A população foi composta por acadêmicos de enfermagem da Instituição de Ensino Superior em Rondônia. A definição da amostra foi de forma não probabilística, proposital ou intencional, do tipo amostragem de variação máxima, em que os pesquisadores escolheram os casos que puderam contribuir mais para as necessidades de informação do estudo, de modo que a investigação não trouxe riscos ou prejuízos para nenhuma das partes envolvidas. A amostra foi obtida através da relação entre os períodos do curso de enfermagem $\left(4^{\circ}\right.$ ao $10^{\circ}$ ), contabilizando 7 (sete) períodos, aos quais foram ofertadas a oportunidade de participação para 3 (três) acadêmicos de cada período, totalizando, 21 (vinte e um) acadêmicos de enfermagem. Os critérios de inclusão foram ser maiores de 18 (dezoito) anos, estar matriculado no referido curso e nos períodos supracitados, terem cursado ou estar cursando a disciplina de Terapia medicamentosa ou disciplina curricular afim, pressupondo de um prévio embasamento teórico.

Diante disso identificamos que apenas dois acadêmicos estavam matriculados no quarto período, por conseguinte, foi necessário adotar o critério de exclusão para um deles, pois, o mesmo ainda não havia cursado a disciplina de terapia medicamentosa. Em consideração a isto, realizamos a coleta com um acadêmico a mais do nono período que se prontificou em participar desta pesquisa e se enquadrou nos critérios de inclusão. 
Utilizou-se um questionário autoaplicável sobre a caracterização dos participantes e questões relacionadas a prática da automedicação. A coleta ocorreu entre os dias 08 (oito) de setembro e 14 (quatorze) de setembro de 2021, após concordarem participar da pesquisa assinando de forma eletrônica o TCLE (Termo de Consentimento Livre e Esclarecido) por meio da plataforma digital Google Forms. Em seguida, cada participante teve acesso ao questionário autoaplicável para responder individualmente, mantendo-se sigilo e privacidade dos dados coletados subsidiado pela Lei Geral de Proteção de Dados (LGPD) nº 13.709 de 2018.

Após a observação da realidade, foi possível identificar as possíveis causas do problema, neste momento foi utilizada a técnica de brainstorming, no intuito de auxiliar na tomada de decisões mediante a solução de um problema. Assim, emergiram os pontos chaves: Alta demanda de automedicação entre os participantes da pesquisa; Autoconfiança na utilização de medicamentos pelo conhecimento prévio.

A partir da análise dos resultados da etapa inicial da coleta de dados, identificou-se pontos-chave e teorização na perspectiva de promover melhora nas situações problemas encontrados, com alternativas viáveis para solucioná-los de maneira crítica e criativa, a partir do confronto entre a teoria e a realidade. Dentre as hipóteses de solução os participantes destacaram: o planejamento de uma intervenção educativa em saúde por meio de uma Oficina Pedagógica e elaboração de um guia de recomendações de boas práticas para prevenção da automedicação. A intervenção elaborada possui uma perspectiva de reflexos resolutivos para os acadêmicos que se automedicam.

Os dados coletados foram estudados com base no método de análise de conteúdo e comparados com os achados da literatura. Os acadêmicos da pesquisa foram identificados pela sigla A1, A2, A3, A4 e assim por diante, no qual o "A" referese a Acadêmico (a) e o numeral a ordem em que foram realizados os questionários, de modo preservar o anonimato dos participantes.

A análise de conteúdo dos dados contida na problematização levantada por meio dos questionários foi submetida à avaliação dos pesquisadores, no qual realizaram a análise das informações relevantes, discutido com o referencial teórico sobre a temática nas seguintes bases de dados Scientific Eletronic Library Online (Scielo), Literatura Latino- América e do Caribe em Ciencias de Saúde (LILACS); Biblioteca Virtual em Saúde (BVS); bem como, na literatura impressa, teses e dissertações.

Por se tratar de uma pesquisa-ação, os participantes envolvidos são levados à construção de novos conhecimentos para transformar a realidade observada. Para este momento realizou-se uma oficina pedagógica no dia 09 (nove) de outubro de 2021, no período vespertino das $15 \mathrm{~h}$ às $16 \mathrm{~h} 30$ minutos, para realização da intervenção de educação em saúde em uma sala de aula ampla da Instituição de Ensino Superior em Rondônia, em que apenas 6 (seis) acadêmicos, de forma voluntária, aceitaram participar do encontro realizado.

Vale ressaltar que nesta etapa, aplicou-se a técnica de roda de conversa, no intuito de instrumentalizar os acadêmicos acerca da temática da pesquisa. Tal técnica contou com os seguintes momentos: 1 - Acolhimento dos participantes da pesquisa: Aplicação de um vídeo de duração curta voltado sobre a temática; 2 - Apresentação dos dados: os pesquisadores explanaram os resultados obtidos na coleta de dados através da identificação do problema. A partir destas informações foi realizado dinâmicas por meio de roda de conversa, com ênfase na farmacovigilância e prevenção de automedicação. 3 Feedback da roda de conversa: obteve-se respostas do aprendizado durante esta etapa na qual a fala dos participantes foi registrada por meio de gravação de voz via celular Iphone 8.

\section{Resultados e Discussão}

Conforme a etapa I do Arco de Charles Maguerez foi realizada a caracterização de 21 (vinte e um) acadêmicos de enfermagem matriculados na instituição de ensino superior. A tabela abaixo traz os resultados desta caracterização dos participantes do estudo. 
Tabela 1. Caracterização dos participantes da pesquisa-ação. Rondônia. 2021.

\begin{tabular}{lcc}
\hline Variável & $\mathbf{N}$ & $\%$ \\
\hline Sexo & 05 & $23,8 \%$ \\
Masculino & 16 & $76,2 \%$ \\
Feminino & \multicolumn{2}{c}{} \\
\hline Faixa Etária & 12 & $57,1 \%$ \\
19 a 25 anos & 04 & $19,1 \%$ \\
26 a 35 anos & 05 & $23,8 \%$ \\
36 a 46 anos & & \\
\hline Cor autodeclarada & 05 & $23,8 \%$ \\
Branco & 12 & $57,1 \%$ \\
Pardo & 04 & $19,1 \%$ \\
Preto & & \\
\hline Turno de estudo & 00 & $0 \%$ \\
Matutino & 00 & $0 \%$ \\
Vespertino & 21 & $100 \%$ \\
Noturno & & \\
\hline
\end{tabular}

Fonte: Vieira, Brumado e Canterle (2021).

Nota-se na Tabela 1 prevalência do sexo feminino, idade de 19 a 25 anos, cor parda e turno de estudo noturno.

O texto de Galvão e Neto (2018) corrobora com os resultados obtidos nesta pesquisa. Na história da Enfermagem há predominância do sexo feminino. Além disso, uma boa parte dos acadêmicos de enfermagem enfrentam dupla jornada de trabalho, tendo como opção realizar o curso no período noturno, pois dependem do seu trabalho para custear a graduação e manter suas famílias. 
Tabela 2. Identificação dos pontos-chaves da pesquisa-ação. Rondônia. 2021.

\begin{tabular}{lll}
\hline Questionário & N & \% \\
\hline Cursou a disciplina de Terapia medicamentosa & & \\
(Farmacologia) & 20 & $95,2 \%$ \\
Sim & 01 & $4,8 \%$ \\
Não & & \\
\hline Uso de medicamentos sem prescrição & 20 & $95,2 \%$ \\
Sim & 01 & $4,8 \%$ \\
Não & & \\
\hline
\end{tabular}

\begin{tabular}{|c|c|c|}
\hline \multicolumn{3}{|l|}{$\begin{array}{l}\text { Sintomas que levam ingerir medicamentos sem } \\
\text { prescrição }\end{array}$} \\
\hline Dores musculares & 11 & $52,4 \%$ \\
\hline Dores de cabeça & 17 & $81,0 \%$ \\
\hline Gripes & 11 & $52,4 \%$ \\
\hline Inflamações & 10 & $47,6 \%$ \\
\hline Tosse & 06 & $28,6 \%$ \\
\hline Azia e dores gástricas & 09 & $42,9 \%$ \\
\hline Febre & 07 & $33,3 \%$ \\
\hline Cólica menstrual & 10 & $47,6 \%$ \\
\hline \multicolumn{3}{|l|}{ Classes de medicamentos mais utilizados } \\
\hline Antitérmicos & 09 & $42,9 \%$ \\
\hline Analgésicos & 18 & $85,7 \%$ \\
\hline Anti-inflamatórios & 13 & $61,9 \%$ \\
\hline Antibióticos & 07 & $33,3 \%$ \\
\hline Anticoncepcionais & 02 & $9,5 \%$ \\
\hline Antialérgicos & 07 & $33,3 \%$ \\
\hline \multicolumn{3}{|l|}{$\begin{array}{l}\text { Motivos que levam a utilizar medicamento sem } \\
\text { prescrição }\end{array}$} \\
\hline Falta de tempo para se consultar & 09 & $42,9 \%$ \\
\hline Os sintomas referidos não requerem consulta médica & 09 & $42,9 \%$ \\
\hline Fácil acesso aos medicamentos & 12 & $57,1 \%$ \\
\hline Dificuldade no acesso para consultas médicas & 05 & $23,8 \%$ \\
\hline \multicolumn{3}{|l|}{$\begin{array}{l}\text { Indicação de medicamentos para familiares, } \\
\text { amigos ou pacientes }\end{array}$} \\
\hline Sim & 15 & $71,4 \%$ \\
\hline Não & 06 & $28,6 \%$ \\
\hline \multicolumn{3}{|l|}{$\begin{array}{l}\text { Autoconfiança em tomar medicamentos sem } \\
\text { prescrição }\end{array}$} \\
\hline Sim & 17 & $81,0 \%$ \\
\hline Não & 04 & $19,0 \%$ \\
\hline \multicolumn{3}{|l|}{ Sabe os riscos que envolvem a automedicação } \\
\hline $\operatorname{Sim}$ & 21 & $100 \%$ \\
\hline Não & 00 & $0 \%$ \\
\hline
\end{tabular}

Fonte: Vieira, Brumado e Canterle (2021).

Para atender ao objetivo do estudo, a partir da identificação do problema, foi realizada uma oficina pedagógica de intervenção como uma forma de educação em saúde. Na oficina pedagógica foram explanados os resultados obtidos através dos questionários via Google Forms.

Foi realizada uma roda de conversa durante a oficina pedagógica, na qual a fala dos participantes foi registrada por meio de gravação de voz. Após a intervenção realizada, as falas que mais se repetiram entre os participantes foram 
selecionadas pelos pesquisadores, gerando três grupos temáticos, a saber: Principais medicamentos utilizados e consequências da automedicação; Automedicação e suas implicações no âmbito jurídico; Autoconfiança dos acadêmicos e a prática de educação em saúde para familiares e amigos.

No Quadro 1, a seguir, foram dispostos os temas levantados seguidos de suas respectivas ideias-chave.

Quadro 1 - Grupos temáticos e ideias-chave levantadas, referente à automedicação entre os acadêmicos de enfermagem. Rondônia, 2021.

\begin{tabular}{|c|l|}
\hline TEMAS LEVANTADOS & \multicolumn{1}{c|}{ IDEIAS-CHAVE } \\
\hline $\begin{array}{c}\text { Principais medicamentos utilizados e consequências da } \\
\text { automedicação }\end{array}$ & $\begin{array}{l}\text { O conhecimento dos participantes sobre a consequência } \\
\text { da automedicação e dos principais medicamentos } \\
\text { utilizados. }\end{array}$ \\
\hline Automedicação e suas implicações no âmbito jurídico & $\begin{array}{l}\text { Relato dos participantes quanto ao desconhecimento do } \\
\text { crime de peculato e das penalidades em casos de furto } \\
\text { de medicamentos. }\end{array}$ \\
\hline $\begin{array}{c}\text { Autoconfiança dos acadêmicos e a prática de } \\
\text { educação em saúde para familiares e amigos }\end{array}$ & $\begin{array}{l}\text { A fala dos participantes quanto a indicação de } \\
\text { medicamentos para terceiros. }\end{array}$ \\
\hline
\end{tabular}

Fonte: Vieira, Brumado e Canterle (2021).

\subsection{Principais medicamentos utilizados e consequências da automedicação}

Quando questionados sobre o que sabiam sobre os principais medicamentos utilizados e suas consequências, a maioria verbalizou que já tinham conhecimento. Notem que A1, A2 e A6 relataram:

\footnotetext{
"Eu sabia que o consumo de medicamentos sem prescrição médica pode causar grande danos à saúde" (A1).

"Os sintomas de dependência e principais medicamentos utilizados na automedicação eu já sabia dos riscos que isso causa a saúde" (A2).
}

"Já sabia sobre as consequências que levam a dependência, sobre os medicamentos como tramal, morfina, etc." (A6).

Encontrou-se nas falas algo preocupante que corrobora com a Tabela 2 (dois), em que todos (100\%) dos participantes que responderam os questionários, afirmam estar cientes e sabem os riscos que envolvem a automedicação e 95,2\% já fez ou faz uso de medicamentos sem prescrição médica, dentre os sintomas que os levam a se automedicarem podemos citar: dores de cabeça $(81 \%)$, dores musculares $(52,4 \%)$, gripes $(52,4 \%)$, inflamações $(47,6 \%)$, cólica menstrual $(47,6 \%)$, azia e dores gástricas $(42,9 \%)$, febre $(33,3 \%)$ e tosse $(28,6 \%)$.

Em um estudo realizado por Souza e Lima (2019) na cidade de Maceió, a prevalência de automedicação entre acadêmicos foi de $93 \%$ afirmando com alto índice de utilização de medicamentos sem prescrição. Está intimamente relacionado com os principais sintomas que afetam a população, os quais são dores musculares, dor de cabeça, gripes, resfriados, azia, cólica abdominal (Sisay et al., 2018).

Dentre os medicamentos assinalados pelos participantes na Tabela 2 (dois), os principais foram analgésicos (85,7\%), anti-inflamatórios (61,9\%), antitérmicos (42,9\%), antibióticos e antialérgicos (33,3\%), anticoncepcionais (9,5\%). A automedicação nessa população foi, sobretudo, motivada por fácil acesso aos medicamentos $(57,1 \%)$, falta de tempo para se consultar (42,9\%), sintomas referidos não requeriam consulta médica $(42,9 \%)$ e dificuldade no acesso as consultas médicas $(23,8 \%)$.

Filler et al. (2020), traz em seu estudo que os medicamentos mais utilizados foram analgésicos/antitérmicos (29,3\%), antialérgicos/anti-histamínicos (14,2\%) e anti-inflamatórios (13,3\%). Sendo motivado por tratamentos anteriores com o mesmo 
medicamento para a mesma doença $(55,6 \%)$, utilização de prescrições anteriores $(49,6 \%)$ e por indicação de amigos e familiares $(39,6 \%)$.

Para Ferreira et al. (2019), os principais fatores que ocasionam a automedicação estão relacionados a comodidade e praticidade, tendo como ideia central a falta de tempo para ir ao médico, tendo como preferência realizar o consumo por conta própria além da facilidade de acesso em farmácias.

A maioria dos medicamentos utilizados pelos acadêmicos são Isentos de Prescrição Médica (MIP), entretanto estes medicamentos devem ser usados de maneira racional, pois seu consumo pode gerar efeitos adversos e comprometer a saúde, podendo levar a morte. Ademais, o tratamento paliativo da dor pode dificultar o diagnóstico da doença e seu tratamento adequado, cronificando seu processo e tornando a experiência mais dolorosa (Menezes, 2021).

\subsection{Automedicação e suas implicações no âmbito jurídico}

Nas falas dos acadêmicos nota-se desconhecimento das consequências, em casos de furto, de medicações no ambiente de trabalho, principalmente aqueles que causam dependência pela automedicação. Somando a isso, além do crime de peculato, o profissional responde pela infração ética cometida nos respectivos conselhos de enfermagem (COFEN/COREN). Ratificando o trecho inicial deste grupo temático, notem as falas de A1, A4 E A6:

\footnotetext{
"Eu não sabia sobre as penalidades jurídicas e do conselho, foi algo que aprendi nesta oficina" (A1).

“Interessante saber sobre o crime de peculato, eu realmente não sabia disso, nem sobre as penalidades" (A4).

"Que os opioides são os mais comuns na abstinência e como são comuns os furtos de medicamentos nos hospitais"' (A6).
}

Os medicamentos opioides mesmo quando administrados por curtos períodos, podem ocasionar a dependência física e psíquica. Essa dependência estimula a busca, geralmente encontrados em ambiente hospitalar, na tentativa de refrear os sintomas de abstinência o indivíduo é levado a automedicar-se de forma compulsiva para alívio da dor, o que pode acarretar prejuízos para sua saúde e comprometer sua vida profissional (Leal, 2020).

Estudo transversal realizado por Barros et al. (2020), descreve que devido a prescrição excessiva de opioides e o fácil acesso aos medicamentos no ambiente hospitalar resulta em uso indevido, abuso e desvio, relacionado ao uso recreativo desses analgésicos. Foi constatado que em 20 países com maior IDH (Índice de Desenvolvimento Humano) no mundo, pontua com um consumo médio de opioides de 216,7mg per capita, o quantitativo de 30,72 vezes maior do que as doses necessárias para o tratamento da dor ocasionado por traumas, câncer e HIV/AIDS.

Nesta perspectiva, devido a dependência e uso descontrolado os profissionais podem chegar a cometer o crime de peculato que está descrito no artigo 312 do Código Penal que tem como objetivo punir o funcionário público que, em razão do cargo, tem a posse de bem público, e se apropria ou desvia o bem, em benefício próprio ou de terceiros. O que prevê uma pena de prisão de 2 a 12 anos e multa.

Fundamentando as consequências do crime de peculato, o indivíduo responde por infração ética no Conselho Federal de Enfermagem (COFEN), que sancionou a Resolução n ${ }^{\circ}$ 564/2017, referente ao Novo Código de Ética dos Profissionais de Enfermagem, conforme as leis têm-se como penalidade a advertência verbal, multa, censura, suspensão de 90 dias do exercício profissional e a mais grave de todas que é a cassação do direito ao exercício profissional por 30 anos. 


\subsection{Autoconfiança dos acadêmicos e a prática de educação em saúde para familiares e amigos}

Verifica-se que 90,5\% já haviam cursado a disciplina de terapia medicamentosa (farmacologia), que está fortemente relacionado com a elevada taxa de autoconfiança em tomar medicamentos sem prescrição e de venda livre, em que $81 \%$ dos acadêmicos confirmam tal dado (tabela 2), o que demonstra estar associado a indicação de medicamentos para familiares, amigos ou pacientes representando $71,4 \%$ da população do estudo.

Rubio et al. (2017) afirmam tais resultados com os dados levantados em sua pesquisa, pois quando os participantes foram questionados se recomendavam os medicamentos a familiares ou amigos, verificou-se que 64,7\% indicaram que sim.

Filler et al (2020), discorrem que o alto nível de conhecimento dos acadêmicos pode estar relacionado com a prática da automedicação, em contrapartida o resultado esperado é oposto para esse grupo, pois o que se espera é que quanto mais conhecimento adquirido, menos são as chances de realizarem práticas deliberadas.

Destaca-se a influência que estes acadêmicos podem ter na sociedade, em que seus conhecimentos sobre os fármacos podem motivar a prática da automedição. Diante disso, observamos que os conhecimentos acerca das medicações são viabilizados após cursarem a disciplina curricular de terapia medicamentosa e afins, gerando assim, autoconfiança para fazer uso e indicações para terceiros.

Quando questionados se repassariam os conhecimentos adquiridos na oficina pedagógica de intervenção, A1 e A3 verbalizaram o seguinte:

"Eu irei repassar os conhecimentos adquiridos para parentes e amigos. Já fiz algumas indicações antes de quais medicamentos tomar e agora entendo o problema nisso" (A1).

"Eu já tive infecção urinária duas vezes e utilizei a prescrição anterior na segunda, porque não conseguir ir ao médico. Mas eu sei que não é o certo. Hoje pude refletir melhor, já fiz indicações para familiares de outros remédios, como por exemplo, dipirona, depois de hoje vou repassar tudo que aprendi aqui" (A3).

A partir das falas supracitadas, foi possível observar que o acadêmico de enfermagem desde a graduação encontra-se inserido no cenário de ensino-aprendizagem, deparando-se com questões oportunas e reais que envolvem a vulnerabilidade social, à saúde e ao ambiente, podendo ser agentes modificadores em suas famílias e comunidades.

O autor Fernandes et al (2019), resgata o papel do enfermeiro como educador em saúde, tanto para os membros da equipe quanto para os seus clientes, com foco em promoção a saúde individual e coletiva. Nesta perspectiva, a graduação deve estar aproximada da realidade, buscando desenvolver nos profissionais uma prática que intervenha e provoque de forma positiva a modificação do cenário de atuação. Somando-se a isso, os profissionais ao assumirem o papel de educadores em saúde, levam a troca de experiência, reflexões, autoanálise das práticas tanto para si mesmos quanto para a comunidade em que se insere.

\section{Oficina Pedagógica de Intervenção: Sensibilização-Ação-Reflexão}

Por se tratar de uma pesquisa-ação com aplicação da oficina pedagógica de intervenção para fortalecer a tríade: sensibilização-ação-reflexão, o start da oficina se deu com os pesquisadores realizando o acolhimento aos participantes da pesquisa. Na ocasião foi entregue a cada participante papel e caneta para anotações de possíveis dúvidas no decorrer da oficina. Logo após, foi apresentado um vídeo curto para introdução a temática do projeto, contendo informações sobre os principais fármacos que causam dependência e as consequências da automedicação. 
No segundo momento da oficina, foram explanados através de slides pelos pesquisadores, os resultados obtidos na coleta de dados seguido de discussões com autores dos seus respectivos estudos sobre a automedicação. Posteriormente foi abordado notícias atualizadas de furto de medicamentos por profissionais em ambiente hospitalar, principalmente as que causam maior dependência, sendo elas, para uso próprio. À vista disso, foi mencionado o desfecho de tal ato, dando ênfase no crime de peculato e penalidades pelo COFEN/COREN pela infração ética cometida.

Diante dos resultados expostos, a oficina foi conduzida por meio de roda de conversa, fluindo de forma dinâmica e expositiva. Em que foi possível reconhecer que a prática educacional é uma forma de capacitar os indivíduos nas tomadas de decisões e os motivando a ter um momento de reflexão, na qual nos possibilitou constatar nas falas dos participantes (A5) e (A2):

\footnotetext{
"Essa pesquisa foi de extrema importância para nossa formação, para não sermos iguais aos profissionais que cometem tal ato. Irei mudar minhas condutas e ser mais consciente quanto a automedicação"' (A5).

"Pude refletir bastante sobre a automedicação. De fato, devemos garantir segurança a nossa saúde" (A2).
}

A execução e dinâmica da oficina pedagógica para educação em saúde, possibilitou liberdade à criação e discussões baseadas em acontecimentos reais, pois além de criar um movimento diferenciado instigou aos participantes da pesquisa uma compreensão mais profunda a respeito do tema, viabilizando a troca de saberes entre estes e os pesquisadores, dispondo-se a contribuir para a sensibilização na perspectiva de reflexos resolutivos para os acadêmicos que se automedicam.

\section{Guia de Boas Práticas para Prevenção da Automedicação}

Para melhor adesão dos acadêmicos, recomenda-se o uso de materiais educativos com impacto positivo na educação em saúde. O guia de recomendações de boas práticas para prevenção da automedicação é um meio eficiente de comunicação para promoção da saúde, levando informações sobre a temática e contribuindo para o esclarecimento de dúvidas (Wachholz et $a l, 2021)$.

Ao utilizar instrumentos educativos são transmitidos saberes técnico-cientifico, desta forma este guia foi desenvolvido como contribuição de enfermagem pelos pesquisadores com o objetivo de orientar os acadêmicos que se automedicam, acerca dos riscos e consequências dessa prática. Visando informar não só ao usuário, mas também toda a família e comunidade. A produção da ferramenta consta layout e ilustrações, com tópicos abordando conceito de automedicação, quais os riscos da automedicação, dependência medicamentosa e síndrome de abstinência, penalidades no âmbito jurídico e como ter boas práticas no consumo de medicamentos.

Para que o conteúdo desse guia de boas práticas seja desenvolvido com sucesso, não basta apenas impor aos acadêmicos que os mesmos sigam todas as orientações que estão exemplificadas no produto. Deve-se sempre praticar a educação em saúde, baseada na tríade: sensibilização-ação-reflexão. Desta maneira, a utilização didática desse método de ensino possibilitará difusão de saberes adquiridos para terceiros, principalmente aos profissionais que já estão atuando na equipe de enfermagem. Afim de que todos encontrem maneiras de melhorar sua qualidade de vida.

\section{Considerações Finais}

Este estudo forneceu informações e conhecimentos relevantes em relação ao fenômeno da automedicação entre acadêmicos de enfermagem em uma Instituição de Ensino Superior em Rondônia, após sua apresentação a esses acadêmicos os 
mesmos demonstraram interesse em suspender a prática da automedicação, além de repassar os conhecimentos adquiridos para familiares e amigos.

Desta forma, mesmo sendo este estudo uma caracterização amostral de uma determinada população, o mesmo destaca o uso indiscriminado de determinadas medicações sem prescrição, este aspecto instiga a reflexão e realização de novos estudos na área, a fim de averiguar tanto a facilidade de acesso a determinadas substâncias, quanto como o conhecimento prévio pode influenciar na prática da ingestão medicamentosa e recomendações a terceiros.

Com a realização deste estudo foi possível contribuir para a academia de enfermagem, reforçando a importância do consumo consciente sob orientação médica, pois os medicamentos podem causar dependência, efeitos adversos e mascarar doenças que podem ser evitadas. A estratégia de intervenção de educação em saúde, por meio da oficina pedagógica proporcionou a conscientização dos acadêmicos de enfermagem através da autoreflexão de condutas frente a automedicação.

\section{Referências}

Alves, D. R. F., Abrantes, G. G. D., Martins, H. K. A., Lima, A. M. C. L., Ramos, F. F. V. R., Santos, A. C. M. D., \& Ribeiro, G. D. S. (2019). Automedicação: prática entre graduandos de enfermagem. Revenferm UFPE, 13(2).

Anvisa, Ministério da Saúde. Instrução Normativa Nº11, de 29 de setembro de 2016.

Barros, G. A. M. D., Calonego, M. A. M., Mendes, R. F., Castro, R. A., Faria, J. F., Trivellato, S. A., \& Dias, A. (2020). Uso de analgésicos e o risco da automedicação em amostra de população urbana: estudo transversal. Revista Brasileira de Anestesiologia, 69, 529-536.

Decreto-Lei Nº 2.848, de 7 de dezembro de 1940 - Código Penal Brasileiro.

Fernandes, J. R., Silva, V. D. C. F., dos Passos Verissimo, W., Vianna, N. T., \& Carneiro, M. L. (2019). Educação em Saúde: o papel do enfermeiro como educador em saúde no cenário de IETC. Revista da Jopic, 2(4).

Ferreira, F. G., de Souza, J. S. M., \& Paim, R. S. P. (2019). Prevalência da Automedicação em Acadêmicos de Enfermagem em uma Faculdade de Caxias do Sul. Revista Contexto \& Saúde, 19(36), 46-52.

Filler, L. N., de Abreu, E. B., da Silva, C. B., da Silva, D. F., \& Montiel, J. M. (2020). Caracterização de uma amostra de jovens e adultos em relação à prática de automedicação. Psicologia e Saúde em debate, 6(2), 415-429.

Galvão, A. P. F. C., \& Neto, A. D. S. S. (2018). A relaçao estresse e hardiness em acadêmicos de enfermagem de uma instituição de ensino superior. JMPHC| Journal of Management \& Primary Health Care| ISSN 2179-6750, 9.

Gama, A. S. M., \& Secoli, S. R. (2017). Automedicação em estudantes de enfermagem do Estado do Amazonas-Brasil. Revista Gaúcha de Enfermagem, 38.

Leal, R. (2020). Uso indevido e de dependência de opioides: da prevenção ao tratamento. Revista de Medicina de Família e Saúde Mental, 2(1).

Lei nº13.709, de 14 de agosto de 2018. Dispõe sobre Lei Geral de Proteção de Dados Pessoais (LGDP). Diário Oficial [da] República Federativa do Brasil, Brasília, DF.

Menezes, A. S. de, Bonanni, I. A., Souza, M. de S. G. C., Carneiro, S. V. G., Alves, S. M., Oliveira, T. A. de, \& Souza, M. B. R. (2021). A automedicação da população mundial: uma revisão integrativa . Research, Society and Development, 10(10), e125101018660.

Pinto, C. D., de Oliveira, N., Silva, R. B. V., Cerdeira, C. D., Garcia, J. A. D., \& Barros, G. B. S. (2021). Automedicação entre estudantes de enfermagem em uma universidade privada no sul de Minas Gerais. Research, Society and Development, 10(8), e25210817129-e25210817129.

RESOLUÇÃO nº 564, de 06 de dezembro de 2017. Dispõe sobre aprovação do novo Código de Ética dos Profissionais de Enfermagem. Cofen - Conselho Federal de Enfermagem.

RIBEIRO, L. D. S., \& Oliveira, C. B. (2018). Automedicação entre estudantes e profissionais de enfermagem.

Rivera, J. G. B., Duarte, F. C. M., da Silva, R. R. C., Monteiro, S. B., Guimarães, M. C. M., \& Vale, V. V. (2021). Impacto da automedicação de fármacos benzodiazepínicos. Brazilian Applied Science Review, 5(4), 1767-1780.

del Toro Rubio, M., Pérez, A. D., Puerta, Z. B., \& Ávila, I. Y. C. (2017). Automedicación y creencias en torno a su práctica en Cartagena, Colombia. Revista cuidarte, 8(1), 1509-1518.

Silva, A. N., de Senna, M. A. A., Teixeira, M. C. B., Lucietto, D. A., \& de Andrade, I. M. (2020). O uso de metodologia ativa no campo das Ciencias Sociais em Saude: relato de experiencia de producao audiovisual por estudantes. Interface: Comunicação Saúde Educação, 24, 1P-1P.

Sisay, M., Mengistu, G., \& Edessa, D. (2018). Epidemiology of self-medication in Ethiopia: a systematic review and meta-analysis of observational studies. BMC Pharmacology and Toxicology, 19(1), 1-12.

Souza, S. N., \& ribeiro de Lima, C. (2019). Automedicação em acadêmicos de enfermagem em uma instituição de ensino superior de Maceió. Caderno de Graduação-Ciências Biológicas e da Saúde-UNIT-ALAGOAS, 5(2), 61. 
Research, Society and Development, v. 10, n. 15, e409101523079, 2021

(CC BY 4.0) | ISSN 2525-3409 | DOI: http://dx.doi.org/10.33448/rsd-v10i15.23079

Tognoli, T. do A., de Oliveira Tavares, V., Ramos, A. P. D., Batigália, F., de Godoy, J. M. P., \& Ramos, R. R. (2019). Automedicação entre acadêmicos de medicina de Fernandópolis-São Paulo. Journal of Health \& Biological Sciences, 7(4 (Out-Dez)), 382-386.

Wachholz, L. F., Knihs, N. D. S., Sens, S., Paim, S. M. S., Magalhães, A. L. P., \& Roza, B. D. A. (2021). Boas Práticas no Cuidado Transicional: continuidade da assistência ao paciente submetido ao transplante de fígado. Revista Brasileira de Enfermagem, 74.

Xavier, C. M., \& da Silva, R. S. (2021). Prevalência da automedicação entre estudantes da universidade federal do sul da Bahia-UFSB-Campus Sosígenes Costa. Visão Acadêmica, 22(1). 